\title{
SMART ASSEMBLY - DATA AND MODEL DRIVEN
}

\author{
${\text { Juhani Heilala*, Heli Helaakoski }{ }^{\S} \text {, Irina Peltomaa }}^{\S}$ \\ *VTT Technical Research Centre of Finland, P.O.Box 1000, 02044 VTT, Finland \\ juhani.heilala@vtt.fi \\ ${ }^{8}$ VTT Technical Research Centre of Finland, P.O.Box 3, 92101 Raahe, Finland \\ heli.helaakoski@vtt.fi, irina.peltomaa@vtt.fi
}

\begin{abstract}
The world is changing distinctly and manufacturing is facing significant challenges. Current manufacturing paradigms need to develop towards better agility to meet current market demand. The relevant research has been launched and the resulting new approaches, like smart manufacturing, digital manufacturing and the cognitive factory, are introduced. However, today's real challenge to manufacturing enterprises is building an infrastructure that will enable the sharing of knowledge and information in a manufacturing environment, regardless of time and place. This article reviews some of the latest approaches and research initiatives and suggests the use of semantic technologies in information integration. Use of semantics enables information and knowledge to be represented in a form understandable to both humans and machines, and offers the ability to adapt more flexibly to change.
\end{abstract}

Keywords smart assembly, semantic interoperability

\section{Introduction}

Today, enterprises are living in a changing business environment characterised by global competition, rapid development of technology, short lead-time, increased cost pressure and more aggressive demand from customers. In Western countries, manufacturing enterprises are not able to compete with labour costs and they need to move on from resource-based manufacturing to knowledge and skill-driven manufacturing. They need to focus on customised high quality, high value added products that require a growing amount of information and knowledge. Today, there is a gap between current market demand and current manufacturing paradigms. There is a need to develop the information integration of the manufacturing process and manufacturing resources towards a smart agile manufacturing process.

The intelligent use of modern ICT as information-producing and communication-enabling technologies has become crucially important. Characteristic of modern ICT is that they significantly increase the capacity of enterprises to efficiently generate and process knowledge-based information [1]. In this paper, "smart manufacturing" is defined as the efficient use of modern ICT, 
including semantic technologies, ubiquitous computing, novel interfaces for humantechnology interaction, human-centred automation, and affordable and flexible robot automation technology in a traditional manufacturing environment. According to Caie [2], the key elements of smart assembly are:

- Empowered, Knowledgeable People: Multi-disciplined, highly skilled workforce empowered to make the best overall decisions.

- Collaboration: People and automation collaboratively working in a safe, shared environment for all tasks.

- Reconfigurable: Modular, plug and play system components easily reconfigured and reprogrammed to accommodate new product, equipment, and software variations and to implement corrections.

- Model and Data Driven: Modelling and simulation tools enabling all designs and design changes to be virtually evaluated, optimized, and validated before being propagated to the physical plant.

- Capable of Learning: Self-integrating and adaptive assembly systems that prevent repeated mistakes and avoid new ones.

The above definition was created in the USA in 2006. The European Technology Platform Manufuture Strategic Research Agenda [3] has similar definitions, with a vision of the manufacturing of the future. According to Westkämper [4], intelligent manufacturing visions are holistic systems, operating in primer fields of high performance and managed by highly skilled workers. They can be adapted by plugand-produce mechanisms and are linked in a digital and virtual engineering and management IT. Adaptive manufacturing recombines new and innovative processes, uses intelligent combinations and flexible configuration of products and manufacturing systems to overcome existing process limitations, and transfers manufacturing know-how using completely new themes or manufacturing-related themes. Adaptive manufacturing takes into account intelligent manufacturing technologies and includes the field of automation and robotics. Adaptive manufacturing also includes new solutions of automation by integration of new methods of cognitive information processing, signal processing and production control by high speed information and communication systems. Intelligent manufacturing can be linked to communications technology networks to assure real-time adaptation. In the future, new technologies like RFID, MES, Wireless, Grid Computing and others will make the vision of a real time factory or smart factory a reality.

A similar approach is the cognitive factory, defined by Zäh et al. [5] as a factory environment with its machines, robots, storages, planning processes and human workers, that is equipped with a sensor network and an IT structure to allow the resources and processes to perceive what they are doing, enable them to control themselves and plan further actions in cooperation with other machines and human workers. The overall goals of the cognitive factory can be summarised as:

- Making the knowledge available in factory systems more transparent

- Enforcing the interconnection between men and machines

- Creating manufacturing systems that are more adaptive than existing ones

- Enabling autonomous planning processes. 
In all the elements shown here, Caie [2], Westkämper [4], Zäh [5], and also Dencker [6] highlight the role of information and knowledge, how information can be accessed and aggregated by both humans and machines. The real challenge for manufacturing enterprises today is to build an infrastructure that enables knowledge and information sharing in a manufacturing environment regardless of time and place. Micro-products are especially challenging given that they might have been designed and developed elsewhere, and that production personnel are facing the challenges. Agility and customer-driven manufacturing increases the complexity of manufacturing operations; the equipment can be complex as well as the products to be assembled.

\section{The role of modern ICT in manufacturing}

One consequence of the deep penetration of ICT into daily life is migration from the conventional factory floor to intelligent manufacturing environments built around the AmI (Ambient Intelligence) paradigm. That is, workplaces with emphasis on greater user-friendliness, more efficient service support, userempowerment, and support for human interaction. Large scale industrial AmI solutions are still rare, even if research efforts are being carried out.

In a manufacturing environment where workforces are surrounded by a collection of reconfigurable production components (physical agents) that include mechatronics, control and intelligence, the challenge is to develop production automation and control systems with autonomy and intelligence capabilities for collaboration, agile and fast adaptation to environmental changes, robustness against the occurrence of disturbances, and easier integration of manufacturing resources and legacy systems.

Today's requirements for high production-process performance, combined with their increasing complexity, represent a real challenge to staff members at all levels (from worker to plant manager) in controlling the production process such that customer orders will be fulfilled to perfection. The concept of digital manufacturing emphasises that the success of manufacturing industries is mainly related to a great diversity and skills of personnel at all levels [4].

The adaptation to changing situations in factory floor operations, in flexible, lean, agile and re-configurable manufacturing, is a challenge. Each product produced can be unique; production personnel must synchronize the material and resources efficiently. The management of information flow is a key issue as shown in Figure 1: 


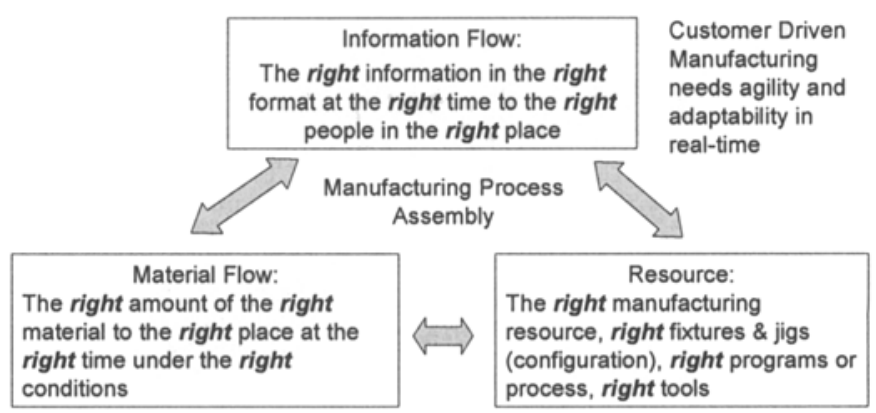

Fig. 1. Information controls material flow and resources

\section{Smart manufacturing}

In the smart manufacturing approach, the human operator interacts and collaborates with a smart manufacturing environment by exploiting modern ICT. Workers have access to aggregated information and knowledge that is supported by an intelligent assistant delivering the relevant piece of information, whenever and wherever it is needed. This will allow workers at different levels, when relevant, to:

- Improve availability of information, communication and knowledge sharing

- Speed up learning of human operators

- Enhance the quality of products, zero defects

- Improve teaching of automated device

- Shorten production standstills, speed of delivery.

Future manufacturing enterprises will be able to capture individual expertise and experience for efficient reuse and draw on a rich, openly accessible shared base of scientific, business, and process knowledge to make informed, accurate decisions and to ensure the right people get the right information at the right time to do their jobs (knowledge-based enterprises). Improved understanding and shared knowledge of the scientific foundations for material and process properties and interactions will support optimized process design and total understanding of complex transformations and interactions at the micro and macro levels.

Collaborative human-centred automation can be seen as automation of a manual task, mechanisation of physical activities and also as cognitive task, computerisation, information and control activities (Fig. 2). This paper focuses on information and control activities and on how to make information available in ICT systems more transparent. 


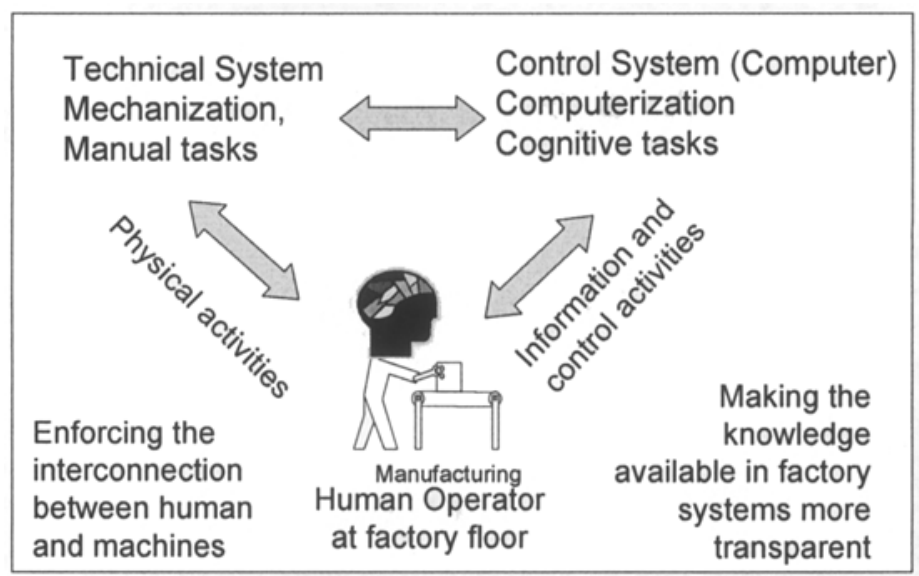

Fig. 2. Collaborative human-centred automation

\section{Digital manufacturing in smart assembly}

Digital manufacturing uses a wide range of engineering and planning tools, software, and ICT to integrate new technologies into manufacturing processes as quickly and as efficiently as possible. The main area of research is the development of integrated tools for industrial engineering and the adaptation of manufacturing, taking into account the configurability of systems. Digital manufacturing is the most important technology of the future. Digital manufacturing needs [4]:

- Distributed data management

- Tools for process engineering

- Tools for presentation and graphic interfaces

- Participative, collaborative and networked engineering

- Interfaces to reality.

Manufacturing systems, processes and data are growing and becoming more complex. Manufacturing engineering and production management decisions involve the consideration of many interdependent factors and variables. These often complex, interdependent factors and variables are probably too many for the human mind to cope with at one time (Fig, 3). For a human decision-maker it is difficult to locate the relevant pieces of information. That is why users on the factory floor, human operators and production managers all need tools for data mining. The challenge of the man-system interface is to bring context-aware data, as a personal advisor, to the operator. 


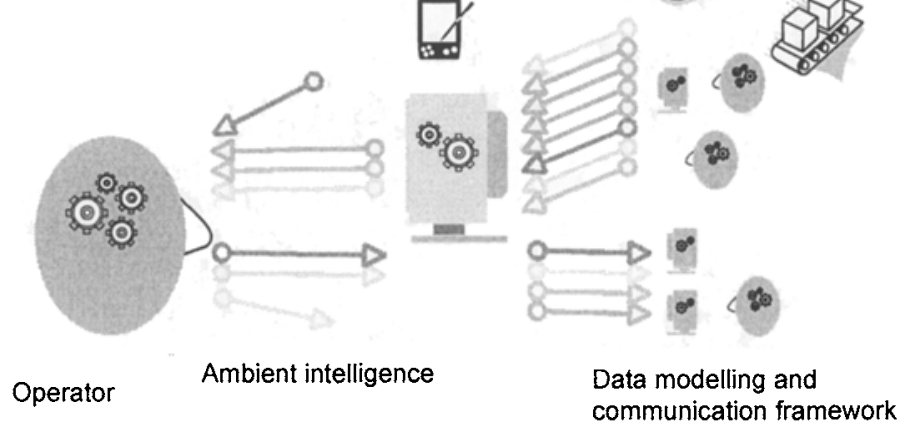

What to show and how to show it: filtering, selecting only relevant information, knowledge sharing and context awareness.

Fig. 3. The amount of information at assembly shops is increasing

Different users require, or are allowed access to, different types of information or the same information presented differently. For instance, a worker, line supervisor and plant manager relies on different levels of data upon which to base decisions too little or too much data and its utility is diminished if not lost. Time is an important factor in defining how much and what kind of data should be aggregated for the various levels of the organization; a manager or worker cannot afford to be "swimming in data" when making a quick decision. Ultimately, users should be given enough information to enable them to make the decisions necessary to optimize the performance of their job function. One of the challenges is to provide the appropriate granularity of information needed by each class of user.

Vision: Integrate human and technical resources to enhance workforce performance, safety and satisfaction, help the human to access "instantaneously" information from a vast array of diverse sources into useful knowledge and effective decisions. Bring into the human-machine system all the skills, rules and knowledge necessary to interpret, diagnose and act in a concerted way between machine knowledge and human intelligence.

Digital manufacturing engineering could be part of the solution. Results presented in a visually attractive way speed up and improve the way they are understood. Virtual reality (VR) and augmented reality (AR) technologies can provide easy to understand visualization. Combined with other production-related tasks AR/VR is an efficient way to create an electronic performance support system (EPSS) for human workers. Examples of how to use AR in assembly work have been given by Sääski et al. [7].

We are able to enable science-based management by workers, if we can provide tools for decision support and information, and the data and models needed in the decision making. Present factory information systems (FIS), like the manufacturing execution system (MES) or automation system capability are geared to support operators and engineers, not planners, financial analysts or upper management. 
Enterprise resource planning (ERP) systems have other limitations; they are usually based on static resource models, with unlimited capacity. Currently, manufacturing scenarios cannot be studied efficiently with the ERP system, at least not by factory floor personnel.

Manufacturers have begun the transition from passive data monitoring to conversion of data to information and must attempt proactive, operational and strategic decision making in the future. Simulation and modelling is one of the tools available for proactive planning. Normally production simulation is used for system design analysis, not yet on the factory floor on-line near real-time. Simulation analysis shows future events with given input parameters. Near real-time simulation is one way to get information for decision making and to create a model-driven smart assembly system.

There has been progress in simulation software, and most of the current software is object-oriented with a graphical windows style user interface. At the same time, however, the complexity in manufacturing systems and products has increased. There are development needs in manufacturing system modelling and simulation, such as faster analysis cycle and automated model building. A simulation model and a manufacturing system life cycle should be combined, and the model should be a virtual, concurrently evolving digital image of the real system. Thus a need exists for real-time data coupling from the factory floor and other manufacturing information systems. A future aim could also be hybrid methods - simulation, optimisation and other manufacturing information systems in an embedded application. These could be used by non-simulation experts, even on-line and realtime on the factory floor (plug and simulate), enabling science-based system management and productivity enhancement tools.

\subsection{Potential in standardised neutral interfaces}

Many standardisation efforts are being made to improve manufacturing system interoperability, but in many cases system integration is still based on custom-built proprietary interfaces. The use of standardised structured manufacturing data in a neutral format (like XML) could clearly increase interoperability between manufacturing information systems. In the digital manufacturing paradigm and use of assembly job shop simulation to improve operations, one of the biggest challenges has been integrating the information system. Work is being done by SISO (Simulation Interoperability Standards Organization (http://www.sisostds.org/)) and CMSD (Product Development Group to create a Core Manufacturing Simulation Data Information Model (CMSDIM)) to standardise a job shop simulation data model. The data elements of the current data model are: Organisations, Calendars, Resources, Skill-definitions, Setupdefinitions, Operation-definitions, Maintenance-definitions, Layout, Parts, Bills-ofmaterials, Inventory, Procurements, Process-plans, Work, Schedules, Revisions, Time-sheets, Probability-distributions, References, and Units-of-measurement [8]. In the future, this development could help lower integration costs, if the SISO CMSD standard is accepted by the industry and by providers of simulation software 
and other manufacturing information systems. The authors believe that suitable standardisation with semantics modelling, and the use of ontologies, are a future solution. For information sharing and integration, semantics technology is clearly one solution, as shown in the next chapter.

\section{Semantics in smart manufacturing}

Manufacturing competitiveness is highly dependant on companies' ability to rapidly reconfigure their manufacturing and assembly systems while the variety of products and volumes is changing rapidly [6]. Over the last decade, several manufacturing concepts and high-level strategies aiming mainly at automation or support of human tasks have been developed [5]. However these concepts alone have not been able to meet the demands of rapid reconfiguration enhanced with increased cost pressure, more aggressive demand from customers and precise product delivery.

To meet the challenges of agile business requirements, future smart manufacturing systems must be able to integrate information and knowledge from disparate sources - humans, machines and task-specific applications - and maintain and share information more effectively than before. The interoperability of information can be achieved through the use of semantics, representing the meaning of data in a way that is understandable to both humans and machines.

Semantic interoperability is defined as a dynamic enterprise capability derived from the application of special software technologies that infer, relate, interpret and classify the implicit meanings of digital content without human involvement, which in turn drive adaptive business processes, enterprise knowledge, business rules and software application interoperability [9]. Semantic interoperability enables the integration of data sources developed using different vocabularies and different perspectives on data. To achieve semantic interoperability, systems must be able to exchange data in such a way that the precise meaning of the data is readily accessible and the data can be translated by any system into a form that it understands [10]. Using a model of a given business domain (ontology) as semantic data, the model can rationalise disparate data sources into one body of information. By creating models for data and content sources and adding generic domain information, integration of disparate sources in the enterprise can be performed without disturbing existing applications. The model is mapped to the data sources (fields, records, files, documents), giving applications direct access to the data through the model of a certain domain.

The concept ontology is defined to be an explicit specification of a conceptualisation [11]. Ontology defines a common vocabulary for information sharing in a domain $[12,13]$ and it includes machine-interpretable definitions of basic concepts in the domain and relations among them [12]. The information infrastructure is created using different ontology architectures and languages. The most efficient languages (RDF [14], OWL [15]) provide semantic and inference support, whereas the lowest level ontology languages like XML [16] only provide information modelling. XML doesn't capture the contextual meaning (or semantics) 


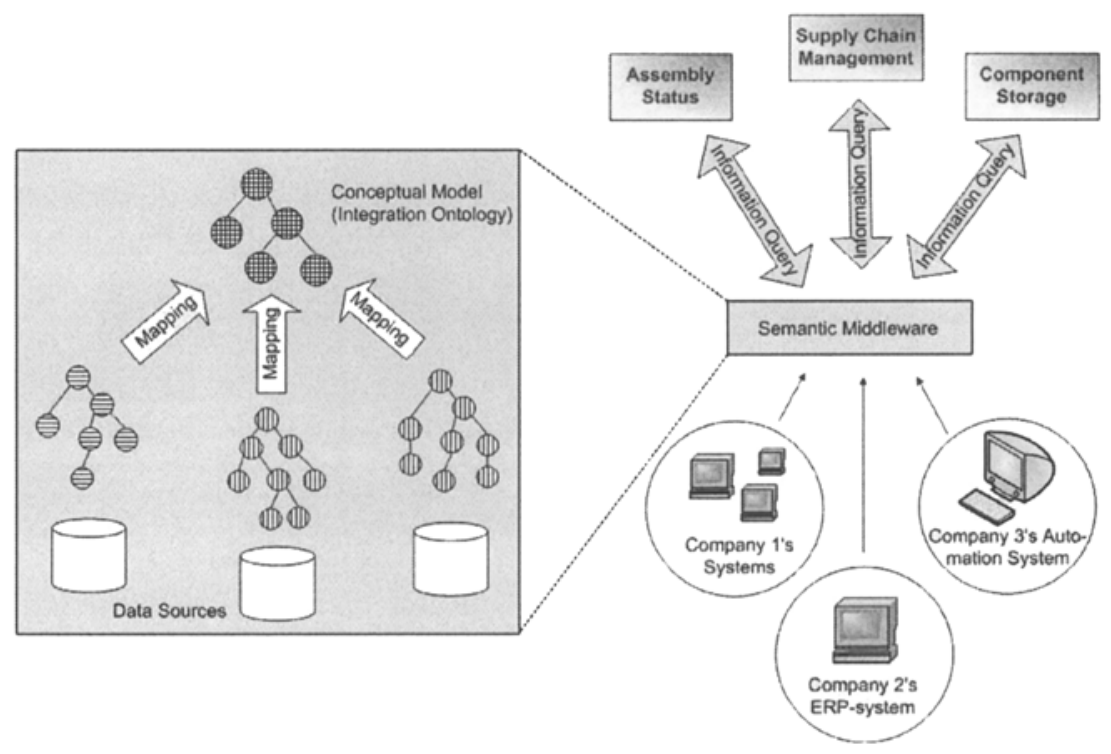

Fig. 4. Through semantic middleware the sharing of mutual information is enabled between a network's companies

of the data since it is a standard for the representation of structured information. The semantics of the XML document is not accessible to machines, only to humans.

The importance of semantics is increasing while companies move away from the traditional view of enterprises as separate, independent entities towards a more collaborative model where the emphasis is on interconnectivity [17]. This development will take over in the near future also in precision assembly. The best knowledge of different expertise fields can be combined together to get competitive advantages in global markets. At this stage the ability to share mutual information and proficiency in interoperability are key assets. All the activities in a company or collaborative networks must be seen as one entity where the same information is advantaged through different applications and through different systems (Fig. 4).

\section{Summary}

In the area of manufacturing there are big challenges to be met. The rapidly changing situation on the factory floor needs to be addressed, the expertise of individual workers needs to be captured, and the vast amount of data needs to be organized. The importance of information and the requirement to share information is recognized in many of the recent approaches presented in this paper: smart assembly, digital manufacturing, the cognitive factory and proactive assembly.

This paper proposes the use of semantic interoperability as an infrastructure that enables information sharing in the manufacturing environment, including assembly. Semantic technologies are used in business process integration but not yet as 
commonly in assembly. Semantic technology is quite a new technology and it is seen as an important technology for supporting interoperability between systems and networks. By bringing together the achievements reached in modern manufacturing approaches with the possibilities of semantic technologies, the challenges of assembly could well be met in the future.

\section{Acknowledgements}

The authors wish to acknowledge the financial support received from VTT. The development is part of the VTT Theme Complex System Design project KNOWMAN - Knowledge and Skill-Driven Manufacturing of High Added-Value Products.

\section{References}

1. M. Castells, Volume 1: The Rise of the Network Society (Blackwell Publishers, Oxford \& Malden, MA, 1996).

2. J. Caie, NIST Workshop Defines Preliminary Roadmaps for Smart Assembly, ARC Advisory Group ARC Strategies, February 2007, p.16; smartassembly.wikispaces.com/space/showimage/NIST+SA+Workshop+SR.doc

3. The Technology Platform on Future Manufacturing Technologies: MANUFUTURE, Strategic Research Agenda, September 2006; http://www.manufuture.org/strategic.html.

4. E. Westkämper, Digital Manufacturing in the global Era, Keynote paper, in: Digital Enterprise Technology, CD-ROM: Proceedings. 3rd CIRP Sponsored Conference on Digital Enterprise Technology, edited by P.F. Cunha (September 18-22, Setubal, Portugal, 2006), p. 11.

5. M.F. Zäh, C. Lau, M. Wiesbeck, M. Ostgathe, and W. Vogl, Towards the Cognitive Factory, in: Proceedings of the 2nd International Conference on Changeable, Agile, Reconfigurable and Virtual Production, CARV 2007 (July 22-24, Toronto, Canada, 2007), pp. 2-16.

6. K. Dencker, J. Stahre, P. Gröndahl, L. Mårtensson, T. Lundholm, and C. Johansson, An Approach to Proactive Assembly Systems - Towards Competitive Assembly Systems, in: Proceedings of the 2007 IEEE International Symposium on Assembly and Manufacturing, ISAM '07 (July 22-25, Ann Arbor, Michigan, USA, 2007), pp. 294-299.

7. J. Sääski, T. Salonen, M. Hakkarainen, S. Siltanen, C. Woodward, J. Lempiäinen. Integration Of Design And Assembly Using Augmented Reality. Fourth International Precision Assembly Seminar, IPAS'2008. Chamonix, France, 10-13 February 2008. Submitted for publication.

8. SISO 2006. Core Manufacturing Simulation Data Information Model, (CMSDIM), PART 1: UML Model. CMSD Product Development Group. Simulation Interoperability Standards Organization. Revision Date: September 13, 2006. www.sisostds.org.

9. J.T. Pollock, and R. Hodgson, Adaptive Information - Improving Business Through Semantic Interoperability, Grid Computing, and Enterprise Integration (John Wiley \& Sons, New Jersey, 2004).

10. S. Ram, and J. Park, Semantic Conflict Resolution Ontology (SCROL): An Ontology for Detecting and Resolving Data and Schema-Level Semantic Conflicts, IEEE Transactions on Knowledge and Data Engineering 16(2), 189-202 (2004). 
11. T. R. Gruber, Towards Principles for the Design of Ontology Used for Knowledge Sharing, Int. Journal of Human-Computer Studies 43(5/6), 907-928 (1995).

12. N.F. Noy, and D.L., McGuinness 2001. Ontology Development 101: A Guide to Creating Your First Ontology, Stanford Knowledge Systems Laboratory Technical Report KSL-0105, March 2001, p. 25; http://protege.stanford.edu/publications/ontology_development/ ontology 101.pdf

13. M. Uschold, and M. Gruninger, Ontologies: Principles, Methods, and Applications, Knowledge Engineering Review 11(2), 93-155 (1996).

14. F. Manola, and E. Miller, RDF Primer, W3C Recommendation, 10 February 2004; http://www.w3.org/TR/rdf-primer/.

15. D.L. McGuinness, and F. van Harmelen, OWL Web Ontology Language - Overview, W3C Recommendation, 10 February 2004; http://www.w3.org/TR/owl-features/.

16. T. Bray, J. Paoli, C.M. Sperberg-McQueen, E. Maler, and F. Yergeau, Extensible Markup Language (XML) 1.0 (Fourth Edition), W3C Recommendation, 16 August 2006; http://www.w3.org/TR/REC-xml/.

17. M. Singh, A Review of the Leading Opinions on the Future of Supply Chains, MIT Center for Transportation \& Logistics Supply Chain 2020 Working Paper, edited by K.

Cottrill, December 2004. p.27;

http://ctl.mit.edu/public/opinions future supply_chains.pdf. 